\title{
The Relation between Concentric and Eccentric Exercise and the Carbohydrate Metabolism in Healthy Individuals*
}

\author{
Aleksandra ZEBROWSKA \\ ${ }^{1}$ Department of Physiology, Academy of Physical Education, Katowice, Poland
}

\section{Abstract}

The purpose of the present study was to determine the response of glucose (G), insulin (In) and insulin-like growth factor (IGF)-I to eccentric (ECC) vs. concentric (CON) resistance exercise in healthy trained and untrained subjects. Venous blood samples were drawn before and immediately after the test, and during 1-hour recovery for the determinations of hormonal and somatomedin concentrations. As compared to resting values, the maximal eccentric (ECC) resistance exercise test caused a significant decrease in G level $(p<0.05)$. Significant differences were observed in post-exercise G level during ECC exercise $(p<0.05)$. Different types of muscle contractions (ECC vs. CON) and resistance training had a significant effect on serum IGF-I concentration at maximal exercise intensity. A significant increase in IGF-I concentration was observed in response to eccentric muscle contraction in trained subjects compared to the control.

Conclusions: These results seem to have demonstrated that in healthy subjects increased serum IGF-I concentration might be responsible for better glucose transport during resistance exercise, and this predominantly in eccentric muscle contraction.

Key words: eccentric exercise, glucose, training, somatomedin

\section{Introduction}

Physical exercise, along with dietary management, constitutes an essential element in blood glucose regulation 6), 7), 15). Regular physical activity positively affects metabolic and cardiovascular functions, and its benefits include enhanced insulin sensitivity, decreased fat mass, improved lipid profile and cardiovascular fitness ${ }^{1), 2}$ ), 12), ${ }^{24)}$. Additionally, all of these metabolic changes play a significant role in the muscle's ability to perform work and prevent the development of metabolic disorders ${ }^{7), 12 \text { ). }}$.

Plasma glucose concentration is a function of the supply of glucose, the glucose transport rate in the circulation, and glucose metabolism ${ }^{7), 17)}$. The glucoregulatory hormones, of which insulin is the most important, help and maintain circulating glucose concentrations in the physiological range. Initially, insulin stimulates the cells of insulin-sensitive tissues, primarily the skeletal muscle, to increase their glucose uptake ${ }^{19), 20)}$. Secondly, insulin acts on the liver to inhibit glucose production ${ }^{2}$. Insulin and muscle contractions induce their stimulatory effect on the glucose transport by translocation of the GLUT4 transporters to the skeletal muscle and adipocytes. The exercise-related mechanism by which GLUT4 is translocated to and from the cell membrane is still not well understood $^{19), 20)}$. Translocation of glucose transporter proteins to cell membrane may be initiated by release of calcium from sarcoplasmic reticulum. It should also be mentioned that recent investigations suggest that the autocrine/paracrine mechanism (e.g., nitric oxide, adenosine, bradykinin, insulin-like growth hormone-I) may exert alternative or parallel actions ${ }^{12)}$, 14), 15). Resistance training stimulates systemic IGF-I production depending on the type, duration, and intensity of exercise. Moreover, sequential concentric (CON) and eccentric (ECC) skeletal muscle contractions are associated with different endocrine responses within the same relative exercise loading ${ }^{5)}$. It has been shown that eccentric contractions cause increased serum IGF-I release in resistance trained athletes $^{27}$. In the study of Yan et al. (1993), serum IGF-I concentrations were higher in eccentric than concentric muscle contractions ${ }^{26)}$.

The beneficial effect of exercise on glycaemic control depends on several factors including type and intensity of exercise, starting levels of glycaemia and insulin secretion ${ }^{6,7)}$. Recent studies have demonstrated that IGF-I and IGF-I binding proteins-3 complex (IGFBP-3) provide an efficient therapeutic option in the prevention of diabetes mellitus ${ }^{3), 4}$. The exercise-related effect of IGF-I secretion on glucose tolerance has been shown in individuals after endurance training ${ }^{9), 10)}$. However, the effect of resistance exercise on insulin and insulin like growth factor-I (IGF-I) concentrations, and especially in different types of muscle contractions, has not been elucidated yet.

It was hypothesized that a stimulus in the form of repetitive resistance exercise with predominantly eccentric skeletal muscle contractions would induce molecular responses which, in combination with IGF-I, might influence circulating glucose concentration in healthy subjects. To test this hypothesis we investigated serum

\footnotetext{
${ }^{*}$ Received 29 January 2010
} 
somatomedin levels and insulin response to eccentric vs. concentric resistance exercise in trained subjects and age-matched controls.

\section{Material and methods}

This study investigated the effect of eccentric (ECC) vs. concentric $(\mathrm{CON})$ resistance exercise on serum glucose (G), insulin (In), insulin like growth factor-I (IGF-I), and insulin like growth factor binding protein-3 (IGFBP-3) concentrations in healthy trained subjects. Ten age-matched, healthy, but untrained men, served as a control group (CG) (Table 1). Trained subjects (RTr) participated in a training protocol consisting of different resistance training regiments (mixed, isometric, and eccentric contractions) during annual training cycle and then participated in arm crank ergometry test. The experimental procedures and possible risks were explained to all participants. The experiment was approved by the Ethics Committee of the Academy of Physical Education in Katowice, and conforms to the standards set by the Declaration of Helsinki.

Following 3 days of standardized normocaloric diet $(37 \mathrm{kcal} / \mathrm{kg} /$ day with $50-60 \%$ carbohydrate, $15-20 \%$ protein, and $20-30 \%$ fat); all subjects underwent bioelectric impedance analysis under resting conditions to measure body fat percentage and total body water. Age, height, body mass, and body mass index (BMI) of the participants (mean \pm SD) are presented in Table 1. All resistance trained and untrained subjects performed graded arm crank ergometry tests predominantly requiring concentric muscle contractions (CON) (shortening of elbow flexors). In the second test the subjects performed an incremental arm exercise with eccentric contractions (ECC) (lengthening of elbow flexors). The subjects were randomly assigned to participation first in CON or ECC exercise. The second test was conducted one week after first one. The tests started with a 3-minute warm-up, and then intensity was increased by $15 \mathrm{~W}$ every 3 minutes up to maximal exercise intensity; $60 \mathrm{rpm}$ were maintained. The tests were performed on a modified ergometer based on a commercial device (Monark, Sweden); the modification allowed setting resistance at CON or ECC modes. Heart rate (HR) was continuously monitored using the PE-3000 Sport-Tester (Polar Inc. Finland). Systolic (SBP) and diastolic blood pressure (DBP) was measured with a sphygmomanometer. Venous blood samples were collected at rest (approximately 15 minutes before exercise) for the assessment of serum insulin-like growth factor (IGF-I), insulin (In), and glucose concentrations. The homeostatic insulin resistance index value was calculated using the following formula proposed by Matthews et al. (1985): HOMAIR = CINS $(\mu \mathrm{IU} / \mathrm{ml}) \times$ CGLUC $(\mathrm{mmol} / \mathrm{l}) / 22.5^{13)}$. The sampling was repeated immediately after cessation and after 1-hour recovery. Serum somatomedin C (ie., IGF-I) was determined in duplicate using DSL ACTIVE IGF-I and IGFBP-3 immunoradiometric assay (IRMA) kits (Diagnostic System Laboratories, Webster, Texas). Serum In was measured with DSL RIA kits (Diagnostic System Laboratories, Webster, Texas. Glucose levels were determined using an enzymatic method (Randox).

Data are presented as means and standard deviation $( \pm \mathrm{SD})$. The data were analyzed with ANOVA test using Statistica 7.1 (StatSoft) software. Intergroup correlation coefficients were determined with Pearson's rank order test. Statistical significance was set at $\mathrm{p}<0.05$.

Table 1. Anthropometric and physiological features of subjects studied.

\begin{tabular}{lcc}
\hline & $\begin{array}{c}\text { Control group }(\mathrm{CG}) \\
(\mathrm{n}=10)\end{array}$ & $\begin{array}{c}\text { Trained subjects }(\mathrm{RTr}) \\
(\mathrm{n}=18)\end{array}$ \\
\hline Age $(\mathrm{yr})$ & $21.4 \pm 0.5$ & $22.4 \pm 1.5$ \\
Body height $(\mathrm{cm})$ & $177.4 \pm 5.9$ & $173.2 \pm 4.3$ \\
Body mass $(\mathrm{kg})$ & $72.1 \pm 5.6$ & $76.5 \pm 5.4$ \\
BMI $(\mathrm{kg} / \mathrm{m} 2)$ & $22.7 \pm 1.0$ & $25.3 \pm 1.6 *$ \\
Body fat $(\%)$ & $12.6 \pm 2.7$ & $14.6 \pm 2.1$ \\
Fat mass $(\mathrm{kg})$ & $9.1 \pm 2.3$ & $11.4 \pm 2.3$ \\
Lean body mass $(\mathrm{kg})$ & $63.0 \pm 4.7$ & $64.6 \pm 4.3$ \\
Total body water(kg) & $46.1 \pm 3.4$ & $52.1 \pm 6.1 *$ \\
VO2max $(\mathrm{ml} / \mathrm{kg} / \mathrm{min})$ & $44.6 \pm 5.5$ & $42.7 \pm 6.2 *$ \\
\hline
\end{tabular}

Data are means $\pm S D$. BMI - body mass index, $V_{2 \max }-$ maximal oxygen uptake

$* p<0.05$ statistical differences between $\mathrm{CG} v s$. RTr

\section{Results}

There was a significant effect of resistance training on basal $(\mathrm{p}<0.01)$ and post-exercise IGF-I concentrations $(\mathrm{p}<0.05)$ (Table 2). Different types of muscle contraction $(\mathrm{ECC}$ vs. CON) had a significant effect on serum IGF-I at maximal exercise intensity $(\mathrm{p}<0.001)$ and after one hour recovery $(\mathrm{p}<0.05)$. Maximal resistance graded CON and ECC exercise tests caused a significant increase in IGF-I level in RTr subjects $(\mathrm{p}<0.001)$ (Fig. 1). Compared to CON exercise, the maximal eccentric (ECC) exercise tests caused a significant increase in IGF-I level $(\mathrm{p}<0.05)$ 
Table 2. Effects of training and type of exercise on insulin-like growth factor (IGF-I), insulin-like growth factor binding protein-3 (IGFBP-3), insulin (In) and glucose (G) concentrations at rest (REST), at maximal intensity of exercise (EX), and after the one-hour recovery (POST EX) in all subjects.

\begin{tabular}{|c|c|c|c|c|}
\hline & \multicolumn{2}{|c|}{$\begin{array}{l}\text { Control group (CG) } \\
\qquad(n=10)\end{array}$} & \multicolumn{2}{|c|}{$\begin{array}{c}\text { Trained subjects (RTr) } \\
(n=18)\end{array}$} \\
\hline & CON & ECC & CON & ECC \\
\hline IGF-IREST $(\mathrm{ng} / \mathrm{mL})$ & $358.3 \pm 57.4$ & $382.0 \pm 38.7$ & $397.1 \pm 42.7$ & $421.6 \pm 62.1 * *$ \\
\hline $\mathrm{IGH}-\mathrm{IEX}(\mathrm{ng} / \mathrm{mL})$ & $444.0 \pm 77.7$ & $413.3 \pm 45.7$ & $449.8 \pm 98.7$ & $499.0 \pm 71.1 * \# \# \#$ \\
\hline IGH-I POST EX $(\mathrm{ng} / \mathrm{mL})$ & $360.3 \pm 54.8$ & $369.3 \pm 36.5$ & $367.0 \pm 53.0$ & $440.0 \pm 82.0 * \#$ \\
\hline IGFBP-3REST (ng/mL) & $6352 \pm 1577$ & $6535 \pm 1703$ & $6468 \pm 1083$ & $6467 \pm 1063$ \\
\hline IGFBP-3EX $(\mathrm{ng} / \mathrm{mL})$ & $6138 \pm 1809$ & $6959 \pm 1909$ & $6908 \pm 1450$ & $7821 \pm 1664 \#$ \\
\hline IGFBP-POST EX $(\mathrm{ng} / \mathrm{mL})$ & $6562 \pm 1189$ & $6687 \pm 1828$ & $7044 \pm 1766$ & $7520 \pm 1048 \#$ \\
\hline $\operatorname{InREST}(\mu \mathrm{IU} / \mathrm{mL})$ & $7.3 \pm 3.2$ & $5.0 \pm 2.3$ & $8.7 \pm 2.4$ & $8.1 \pm 0.9 *$ \\
\hline $\operatorname{InEX}(\mu \mathrm{IU} / \mathrm{mL})$ & $10.0 \pm 3.4$ & $8.0 \pm 2.9$ & $10.3 \pm 4.0$ & $9.2 \pm 2.8$ \\
\hline $\operatorname{InPOST} \operatorname{EX}(\mu \mathrm{IU} / \mathrm{mL})$ & $7.8 \pm 4.3$ & $8.7 \pm 5.2$ & $10.1 \pm 3.7$ & $8.7 \pm 3.1 \#$ \\
\hline GREST (mmoL/L) & $5.4 \pm 0.5$ & $5.2 \pm 0.4$ & $5.5 \pm 0.5$ & $4.9 \pm 0.2$ \\
\hline GEX (mmoL/L) & $5.7 \pm 0.9$ & $5.5 \pm 0.3$ & $6.0 \pm 0.9$ & $5.3 \pm 0.4$ \\
\hline GPOST EX (mmoL/L) & $5.5 \pm 0.7$ & $5.4 \pm 0.3$ & $5.8 \pm 0.4$ & $5.2 \pm 0.2 \# \# \#$ \\
\hline
\end{tabular}

Data are means \pm SD. $* p<0.05, * * p<0.01$ for the effect of training $v s$. the corresponding control group; ${ }^{*} p<0.05$,

$\#^{\#}<0.001$ for the tvpe of exercise. ECC $v s$. CON. in either group of subiects.

(Fig. 1). The post-exercise concentrations of serum IGF-I were significantly higher in trained subjects compared to CG but only in ECC exercise test $(\mathrm{p}<0.05)$ (Fig. 1). A significant effect of training was observed in resting insulin concentration $(\mathrm{p}<0.05)$. However, no significant difference was seen in glucose levels $(\mathrm{G})(\mathrm{p}>0.05)$. Different types of muscle contraction (ECC vs. CON) had a significant effect on serum IGFBP-3 at maximal exercise intensity $(\mathrm{p}<0.05)$ and during 1 -hour recovery $(\mathrm{p}<0.05)$. Significant effects of CON and ECC exercise were observed regarding serum insulin $(\mathrm{p}<0.05)$, and glucose concentration $(\mathrm{p}<0.001)$ during one-hour recovery (Table 2).

When analyzed by groups, RTr subjects had significant higher power output in CON exercise compared to CG $(153.3 \pm 19.6$ vs. $109.5 \pm 18.8 \mathrm{~W} . \mathrm{p}<0.001)$ and in ECC exercise compared to CG $(154.7 \pm 28.1 \mathrm{~W}$ vs. $128.0 \pm 14.6$; $\mathrm{p}<0.05)$. Eccentric muscle contraction (ECC) significantly increased serum IGFBP-3 concentration in RTr group when compared to $\mathrm{CON}$ exercise $(\mathrm{p}<0.05)$ (Fig. 2).Compared to resting values, the maximal eccentric (ECC) resistance exercise test resulted in a significant decrease in glucose $(G)$ levels $(p<0.05)$ (Fig. 3). Significant differences were also seen regarding $G$ concentrations after 1-hour recovery following ECC exercise $(p<0.01)$. No significant influence of exercise was observed in insulin concentrations after different types of muscle action (Fig. 4). A tendency toward higher serum In was revealed after CON exercise in all groups under investigation ( $\mathrm{p}>0.05)$. A significant increase in insulin resistance index (HOMAIR) was only seen at rest in RTr subjects compared to CG (Fig. 5). Significant negative correlations were seen between post-ECC IGF-I levels and insulin, $\mathrm{p}<0.05$ ( $\mathrm{r}=-$ 0.31). There was also a correlation between basal glucose and IGF-I levels $\mathrm{p}<0.05(\mathrm{r}=-0.27)$, and post-ECC glucose and IGF-I concentrations $\mathrm{p}<0.001(\mathrm{r}=-0.48)$.

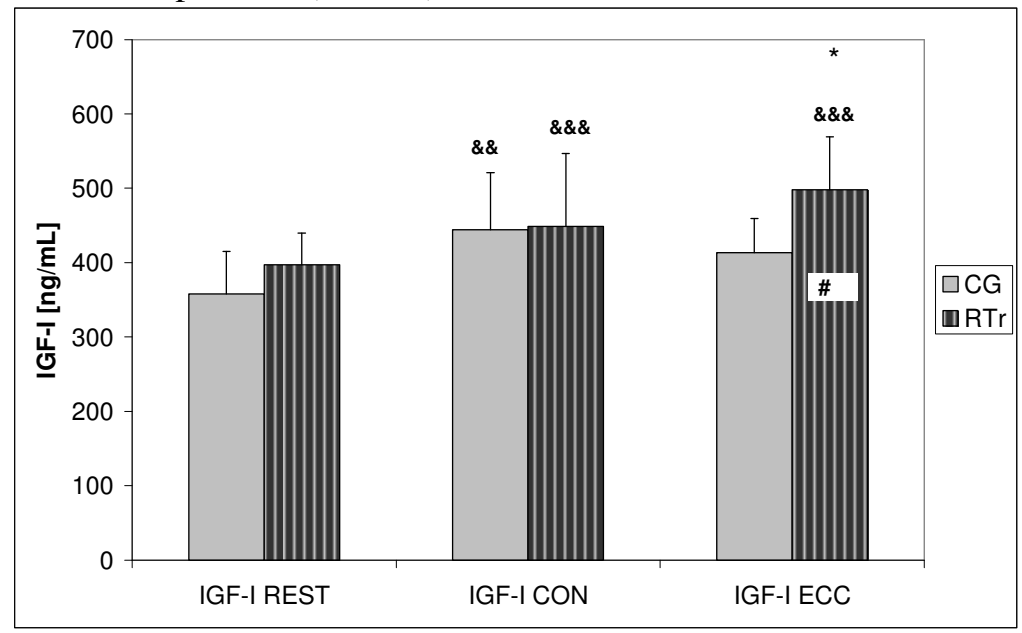

Fig.1. Insulin like growth factor-I (IGF-I) concentration before (rest) and at maximal intensity of CON and ECC exercise in control group $(\mathrm{CG})$ and resistance trained subjects $(\mathrm{RTr}){ }^{\& \&} p<0.01,{ }^{\& \& \&} p<0.001$ significant differences between rest concentrations and at maximal intensity of CON or ECC exercise; ${ }^{*} p<0.05$ significant differences between CON and ECC exercise $* p<0.05$ significant differences between control group (CG) and resistance trained subjects (RTr). 


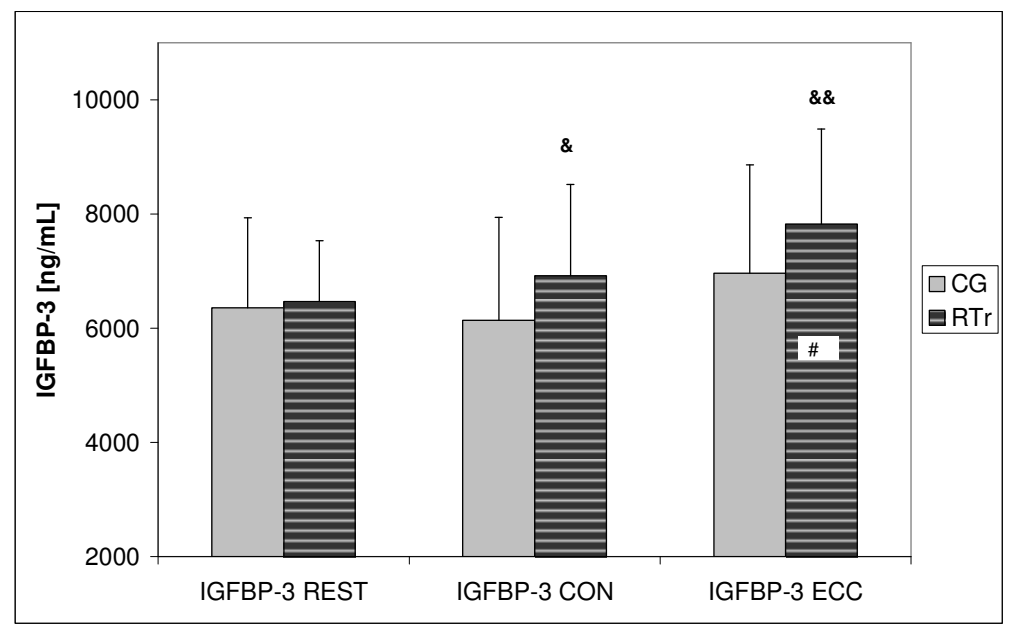

Fig.2. Insulin like growth factor binding protein-3 (IGFBP-3) concentration before (rest) and at maximal intensity of CON and ECC exercise in control group (CG) and resistance trained subjects (RTr). ${ }^{\&} p<0.05,{ }^{\& \&} p<0.01$ significant differences between rest concentrations and at maximal intensity of CON or ECC exercise; ${ }^{\#} p<0.05$ significant differences between CON and ECC exercise.

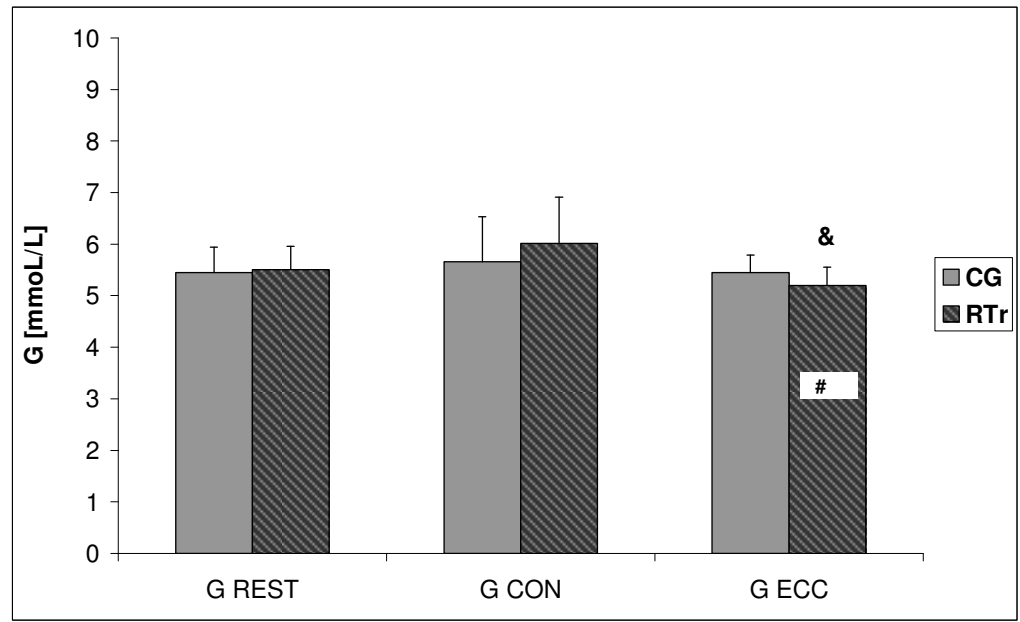

Fig.3. Glucose concentration before (rest) and at maximal intensity of CON and ECC exercise in control group (CG) and resistance trained subjects $(\mathrm{RTr}) .{ }^{\&} p<0.05$ significant differences between rest concentrations and at maximal intensity of CON or ECC exercise; ${ }^{\#} p<0.05$ significant differences between CON and ECC exercise.

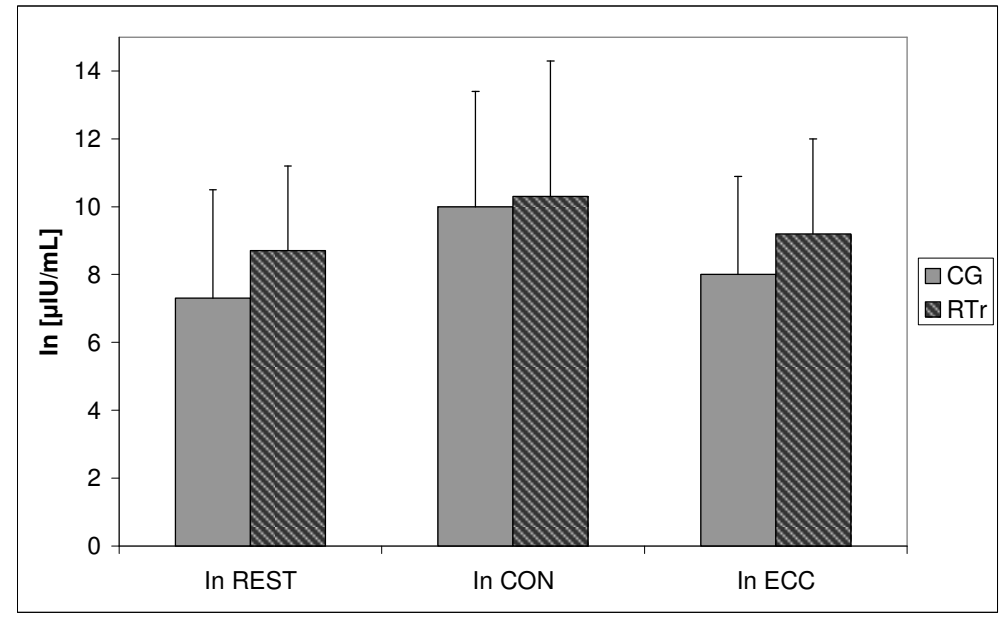

Fig.4. Insulin concentration before (rest) and at maximal intensity of CON and ECC exercise in control group (CG) and resistance trained subjects $(\mathrm{RTr})$. 


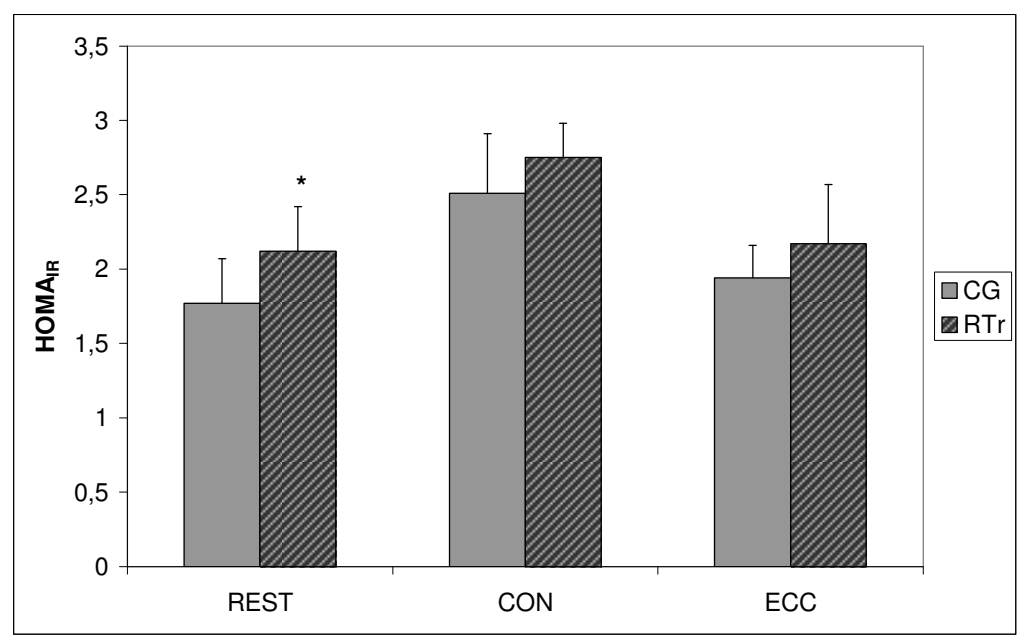

Fig.5. Insulin resistance index $\left(\mathrm{HOMA}_{\mathrm{IR}}\right)$ before (rest) and at maximal intensity of CON and ECC exercise in control group $(\mathrm{CG})$ and resistance trained subjects (RTr). ${ }^{*} p<0.05$ significant differences between control group (CG) and resistance trained subjects $(\mathrm{RTr})$.

\section{Discussion}

The purpose of the present study was to determine the response of glucose, insulin, insulin-like growth factor (IGF-I), and IGF-I binding proteins -3 (IGFBP-3) to resistance training. The effect of eccentric vs. concentric exercises on glucose concentration was also investigated. Despite the identical exercise load during training protocols, the results of concentric and eccentric exercise tests demonstrated some differences in glucose and IGFI, IGFBP-3 concentrations in resistance trained subjects. We hypothesize that a considerable increase of blood glucose utilization during and after ECC exercise might have been caused by exercise related changes in serum somatomedin (IGF-I) levels. Such a hypothesis seems to be lent support by the fact that serum $\mathrm{G}$ was decreased in resistance ECC, but not CON, exercise in trained subjects. Moreover, higher IGF-I/IGFBP-3 level after ECC compared to $\mathrm{CON}$ loading seems to suggest a beneficial effect of resistance exercise with eccentric muscle contraction on glycaemia control.

Several mechanisms of glucose variations following exposure to exercise in healthy subjects have been proposed $^{20), 21), 23)}$. Muscle contraction increases insulin sensitivity and stimulates the muscular uptake of glucose $^{20), 25)}$. Since glucose uptake during muscle contraction has been observed in the absence of insulin, the role of an insulin-independent pathways have been suggested ${ }^{14)}$. Manetta et al. (2003) demonstrated that endurance training in middle-aged men increased the activity of the GH/IGF-I system and improved glucoregulation both at rest and during high-intensity endurance exercise ${ }^{9)}$. The beneficial effect of combined aerobic and resistance exercise on glycaemic control and increase expression and translocation of GLUT-4 glucotransporters and their coding mRNA was also documented ${ }^{6)}$. Other studies have demonstrated that programmes combining some forms of both endurance and resistance exercise, and high - force eccentric resistance exercise can lead to significant improvements in glycaemic control ${ }^{11), 12), 24)}$. However, the benefits of resistance exercise are not limited to enhanced glucose regulation; muscular strength, lean tissues mass and power are also increased ${ }^{5), 8), 24)}$. Eccentric muscle contractions induce greater force per unit of muscle compared to concentric load, and therefore constitute a potent stimulus for skeletal muscle hypertrophy; high force production at low energetic cost associated with eccentric exercise may be beneficial to individuals with lower exercise tolerance ${ }^{8), 11)}$.

It has been documented that both types of muscle contraction during resistance training have significant effects

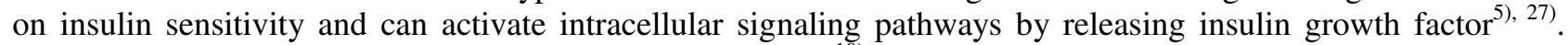
Somatomedin, in turn, increases the transport of glucose ${ }^{10)}$, and may be involved in the growth-promoting responses $^{5)}$. Serum somatomedin appears to be inversely related to insulin levels. Insulin-like growth factor-I and its binding proteins (IGFBPs) may modulate insulin sensitivity and insulin-independent transport of glucose ${ }^{9), 10)}$. IGFBP-1 and IGFBP-3 are the most important of the 6 circulating IGFBPs ${ }^{22}$. Although, IGFBP-1 play a role in glucoregulatory process, the level of circulating IGF-I are mediated predominantly by IGFBP-3, which may be responsible for better delivery of IGF-I to the cells in both trained and untrained subjects ${ }^{9), 22)}$. Our investigations revealed a significant increase in IGF-I concentrations following CON and ECC exercise in trained subjects. The maximal resistance graded ECC exercise test led to a significant increase in IGF-I level and decreased glucose concentration compared to concentric exercise. Negative correlations between IGF-I level and glucose and insulin levels also seem to suggest that a serum IGF-I increase in response to repeated bouts of acute high-intensity eccentric exercise during resistance training may act as a causative factor resulting in higher glucose uptake. 
An increase in post-ECC glucose transport to muscle cells has also been reported ${ }^{11), 27)}$. The precise mechanism underlying such response remains unknown, but cell membrane repair after intensive eccentric exercise might require a significant amount of blood glucose. Apart from differences in muscle contractions, increased glucose transport might also be associated with differences in glucose clearance between oxidative and glycolytic muscle fibers during arm and leg muscle contractions ${ }^{16}$. Our results suggest that increases in glucose sensitivity and strength-related performance parameters could be greater following an exercise programme consisting of maximum eccentric muscle actions than after resistance training using concentric muscle actions only. We conclude that ECC exercise is the most effective for glucose transport independent of the subject's training status. In healthy subjects, energy demand and heart rate responses to eccentric exercise are small relative to the amount of muscle force produced. Thus, eccentric exercise may be an attractive alternative to resistance exercise for patients with metabolic disorders and limited cardiovascular exercise tolerance.

In conclusion, this study shows that repetitive resistance exercise with predominantly eccentric skeletal muscle contractions induced molecular responses which, in combination with IGF-I, might increase insulin-independent glucose transport to muscle cells. These results imply that in patients with glucose metabolism disturbances or diabetes a combination of aerobic exercise with graded eccentric resistance training may help regulate glucose control without an excessive load to the cardiovascular system.

\section{References}

1) Blair S.N. Exercise, successful aging, and disease prevention and weight control. (In :) Exercise physiology. Energy nutrition \& human performance. W.D. McArdle., F.I. Katch, V.L. Katch (ed.) Lippincott Williams \& Wilkins Philadelphia, 2007; 883-903

2) Camacho R.C., Galassetti P., Davis S.N., Wasserman D.H. Glucoregulation during and after exercise in healthy and insulin dependent diabetes. Exerc. Sport Sci. Rev. 2005; 33: 17-23.

3) Chen W., Salojin KV., Mi Q., Grattan M., Meagher T.C., Zucker P., Delovitch T.L. Insulin-like growth factor (IGF-)I/IGF-binding protein complex: therapeutic efficacy and mechanism of protection against type 1 diabetes. Endocrinol. 2004; 145: 627-638.

4) Clemmons D.R., Moses A.C., McKay M.J., Sommer A., Rosen D.M., Ruckle J. The combination of insulin-like growth factor I and insulin-like growth factor binding protein-3 reduces insulin requirements in insulin-dependent type 1 diabetes: evidence for in vivo biological activity. J Clin Endocrinol. Metab. 2000; 85: 1518-1524.

5) Hameed M., Orrell R.W., Cobbold M., Goldspink G., Harridge S.D. Expression of IGF-I splice variants in young and old human skeletal muscle after high resistance exercise. J. Physiol. 2003; 547: 247-254.

6) Hawley J. A., Lessard S.J. Exercise training-induced improvements in insulin action. Acta Physiol (Oxf.), 2008; 192: 127-135.

7) Holoszy J.O. Exercise-induced increase in muscle insulin sensitivity. J Appl Physiol. 2005; 99: 338-343.

8) Lindstedt S.L., LaStayo P.C., Reich T.E.: When active muscles lengthen: properties and consequences of eccentric contractions. News Physiol. Sci. 2001; 16: 256-261.

9) Manetta J., Brun J.F., Fedou C., Maïmoun L., Prefaut C., Mercier J. Serum levels of insulin-like growth factor-I (IGF-I), and IGF-binding proteins-1 and-3 in middle-aged and young athletes versus sedentary men: relationship with glucose disposal. Metabolism. 2003; 52: 821-826.

10) Manetta, J. Brun, J.F. Maïmoun, L. Callis, A. Prefaut C., Mercier J. Effect of training on the GH/IGF-I axis during exercise in middle-aged men: relationship to glucose homeostasis. Am. J. Physiol. Endocrinol. 2002; 283: 929-936.

11) Marcus R.L., Smith S., Morrell G., Addison O., Dibble L.E., Wahoff-Stice D., LaStayo P. Comparison of combined aerobic and high - force eccentric resistance exercise with aerobic exercise only for people with type 2 diabetes mellitus. Phys. Ther. 2008; 11: 1345-1354.

12) Marliss E.B., Vranic M. Intense exercise has unique effects on both insulin release and its role in glucoregulation. Diabetes. 2002; 51: 271-283.

13) Matthews D.R., Hosker J.P., Rudenski A.S., Naylor B.A., Treacher D.F., Turner R.C. Homeostasis model assessment: insulin resistance and beta-cell function from fasting plasma glucose and insulin concentrations in man. Diabetes. 1985; 28: 412-419.

14) McGee S.L., Hargreaves M. Exercise and skeletal muscle glucose transporter 4 expression: molecular mechanisms. Clin Exp Pharmacol Physiol. 2006; 33: 395-399.

15) Mikines K.J., Sonne B., Farrell P.A., et al. Effect of physical exercise on sensitivity and responsiveness to insulin in humans. Am J Physiol. 1988; 254: 248-259.

16) Olsen D.B, Sacchetti M., Dela F., Ploug T., Saltin B. Glucose clearance is higher in arm than leg muscle in type 2 diabetes. J. Physiol. 2005; 565: 555-562. 
17) O'Reilly J., Wong S.H.S., Chen Y. Glycaemic index, glycaemic load and exercise performance. Sports Med. 2010; 40: 27-39.

18) Rajaram S., Baylink D.J., Mohan S.: Insulin-like growth factor-binding proteins in serum and other biological fluids: regulation and functions. Endocr Rev. 1997; 18: 801-831.

19) Richter E.A., Nielsen J.N., Jorgensen S.B., Frosig C., and Wojtaszewski J.F.P.: Signaling to glucose transport in skeletal muscle during exercise. Acta Physiol. Scand. 2003; 178: 329-335.

20) Sakamoto K., Goodyear L.J. Invited review: intracellular signaling in contracting skeletal muscle. J Appl Physiol. 2002; 93: 369-383.

21) Santos J.M., Ribeiro S.B., Gaya A.R., Appell H.J., Duarte J.A. Skeletal muscle pathways of concentration-enhanced glucose uptake. Int J Sports Med. 2008; 29: 785-794.

22) Schwarz A.J., Brasel J.A., Hintz R.L., Mohan S., Cooper D.M.: Acute effect of brief low- and highintensity exercise on circulating insulin-like growth factor (IGF) I, II, and IGF-binding protein-3 and its proteolysis in young healthy men. J Clin Endocrinol Metab. 1996; 81: 3492-3497.

23) Thong F.S., Bilan P.J., Klip A.: The Rab GTPase-actvating protein AS160 integrates Akt, protein kinase C, and AMP-activated protein kinase signals regulating GLUT-4 traffic. Diabetes. 2007; 56: 414-423.

24) Turcotte L.P., Fisher J.S. Skeletal muscle insulin resistance: roles of fatty acid metabolism and exercise. Phys. Ther. 2008; 88: 1279-1296.

25) Wojtaszewski J.F.P., Richter E.A.: Glucose utylizaton during exercise: influence of endurance training. Acta Physiol. Scand. 1998; 162: 351-358.

26) Yan, Z., Biggs R.B., Booth F.W.: Insulin-like growth factor immunoreactivity increases in muscle after acute eccentric contractions. J. Appl. Physiol. 1993; 74: 410-414.

27) Zebrowska A., Zajac A., Poprzecki S., Waligora G.: The influence of eccentric and concentric muscle contractions on growth factors in strength trained athletes. Journal of Human Kinetics 2005; 13: 17-26.

Corresponding author:

Aleksandra Zebrowska, Ph.D.

Department of Physiology

Academy of Physical Education

Mikolowska st. 72A

40-065 Katowice, Poland

E-mail: olazebrowska@yahoo.com

Tel.:+48 322075145

Fax: +48322075200 